\title{
TALCOSE PULMONAR ASSOCIADA AO USO ENDOVENOSO DE MEDICAMENTOS ORAIS: RELATO DE CASO*
}

\author{
Arthur Soares Souza Jr. ${ }^{1}$, Edson Marchiori' ${ }^{2}, K_{\text {Klaus L. Irion }}{ }^{3}$, Ângela Ferreira ${ }^{4}$, Melissa Koch $^{5}$
}

Resumo Neste trabalho são apresentados os achados observados na tomografia computadorizada de alta resolução de um paciente com talcose pulmonar, com a doença adquirida pelo uso de drogas orais injetadas por via venosa. 0 principal aspecto observado na tomografia computadorizada de alta resolução foi o de nódulos centrolobulares, associados a massas conglomeradas. Havia também enfisema e áreas de atenuação em vidro fosco. Estes achados são bastante sugestivos de talcose pulmonar.

Unitermos: Talcose; Tomografia computadorizada de alta resolução; Doença pulmonar.

Abstract Pulmonary talcosis associated with intravenous use of oral medications: a case report.

We report the findings of the high-resolution computed tomography of the chest of a patient with pulmonary talcosis related to intravenous injection of diluted oral medicine. The most important high-resolution computed tomography findings were small centrilobular nodules associated with conglomerated masses. Areas of emphysema and ground glass attenuation were also seen. These high-resolution computed tomography findings are highly suggestive of pulmonary talcosis.

Key words: Talcosis; High-resolution computed tomography; Lung disease.

\section{INTRODUÇÃO}

O talco (silicato de magnésio) é um mineral amplamente usado em várias indústrias, como cerâmica, papel, plásticos, borracha, pintura, construção e cosmética, entre outras ${ }^{(1)}$.

São reconhecidas quatro diferentes formas de doença pulmonar causada pelo talco. Três delas estão relacionadas à aspiração e se diferenciam, entre si, pela composição do pó aspirado. Assim, o talco aspirado pode ser puro (talcose), estar associado a partículas de sílica (talcossilicose), ou a fibras de asbesto (talcoasbestose). A úl-

* Trabalho realizado no Serviço de Radiodiagnóstico do Hospital Universitário Clementino Fraga Filho (HUCFF) da Universidade Federal do Rio de Janeiro (UFRJ), Rio de Janeiro, RJ, e no Serviço de Radiologia do Hospital de Base da Faculdade de Medicina de São José do Rio Preto (Famerp), São José do Rio Preto, SP.

1. Professor Adjunto de Radiologia da Famerp, Coordenador de Pós-Graduação do Instituto de Radiodiagnóstico Rio Preto.

2. Professor Titular de Radiologia da Universidade Federal Fluminense (UFF), Coordenador Adjunto do Curso de Pós-Graduação em Radiologia da UFRJ.

3. Radiologista do Pavilhão Pereira Filho do Complexo Hospitalar Santa Casa de Porto Alegre e da Irion Radiologia, Douto em Medicina pela Universidade Federal do Rio Grande do Su (UFRGS).

4. Professora Adjunta de Pneumologia da UFF, Doutora em Medicina pela UFRGS.

5. Médica Pós-graduanda em Radiologia na Santa Casa de Misericórdia do Rio de Janeiro.

Endereço para correspondência: Prof. Dr. Edson Marchiori. Rua Thomaz Cameron, 438, Valparaíso. Petrópolis, RJ, 25685-120. E-mail: edmarchiori@zipmail.com.br

Recebido para publicação em 26/12/2002. Aceito, após revisão, em 7/1/2003. tima forma é aquela devida à administração do talco por via endovenosa ${ }^{(2)}$.

A radiologia, particularmente a tomografia computadorizada de alta resolução (TCAR), desempenha papel importante na avaliação de pacientes usuários de drogas injetáveis com sintomas respiratórios, especialmente os portadores da síndrome da imunodeficiência adquirida (SIDA), quando os aspectos tomográficos muitas vezes servem para diferenciar as lesões secundárias à injeção de talco das infecções ou neoplasias associadas à SIDA.

\section{RELATO DO CASO}

Paciente do sexo masculino, de 33 anos de idade, com queixas de dispnéia progressiva e tosse seca. Fumante de cerca de 30 cigarros por dia. Relatava fazer uso de drogas injetáveis, macerando comprimidos de Provigil $^{\circledR}$ (modafinil - droga estimulante do sistema nervoso central).

Foi realizada TCAR, que mostrou pequenos nódulos centrolobulares, com atenuação em vidro fosco, mais bem identificados nos campos superiores. Havia também áreas de confluência, com massas conglomeradas, com extenso grau de enfisema, predominando nos campos inferiores e adjacente às massas (Figura 1). Nas janelas para mediastino eram observadas áreas de aumento de densidade, amorfas, irregulares, no interior das massas conglomeradas (Figura 2).

\section{DISCUSSÃO}

Mais de 500 diferentes produtos são vendidos sob a designação de talco ${ }^{(\mathbf{1})}$. O termo é usado tanto para o silicato de magnésio puro ${ }^{(3,4)}$ como para uma mistura de minerais contendo a substância pura e diversos outros produtos ${ }^{(2)}$. Por esta variabilidade de composição, o padrão da doença pulmonar associada com a inalação de talco pode ser muito variável, e achados clínicos, radiológicos e anatomopatológicos atribuídos ao talco freqüentemente se devem a outros componentes da mistura, especialmente a sílica e o asbesto ${ }^{(\mathbf{1}, \mathbf{2}, \mathbf{5})}$.

Pneumoconiose por talco já foi descrita em pessoas que trabalhavam em minas de talco (extração), na moagem (pulverização), no embalamento e no transporte do produto, trabalhadores com pedra-sabão, que contém talco, sílica e asbesto, e trabalhadores que usam o talco no processo de produção, como na indústria da borracha, papel, produtos têxteis, couro, cerâmicas, produtos farmacêuticos, cosméticos, inseticidas, herbicidas, e outros ${ }^{(1,3,5-7)}$

Outra forma de exposição é a utilização de medicamentos feitos para uso oral injetados por via endovenosa. Alguns usuários de drogas habitualmente maceram 


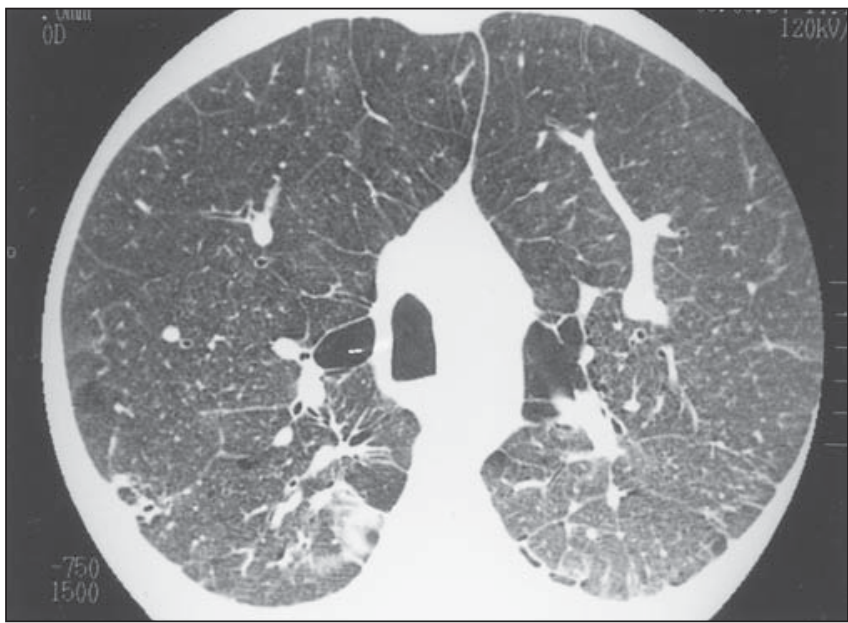

A

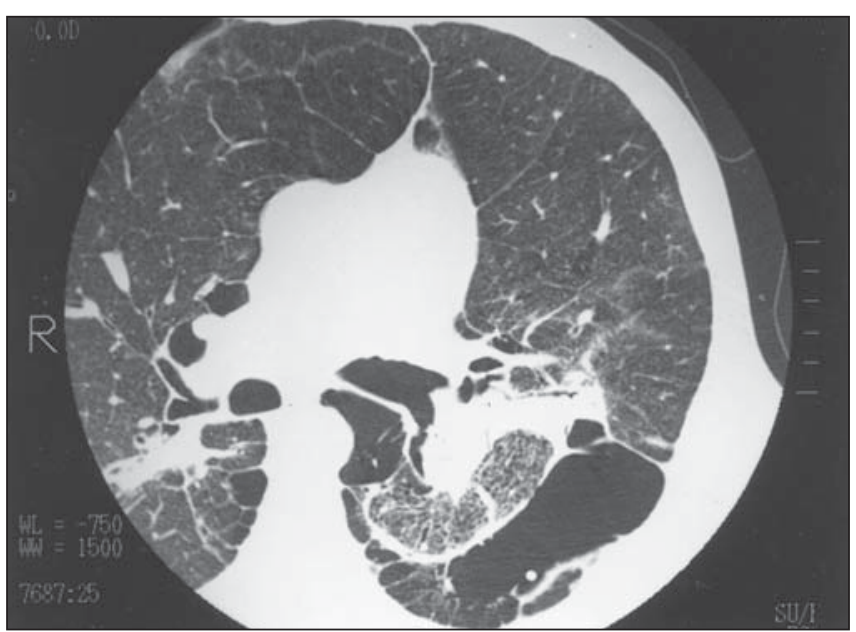

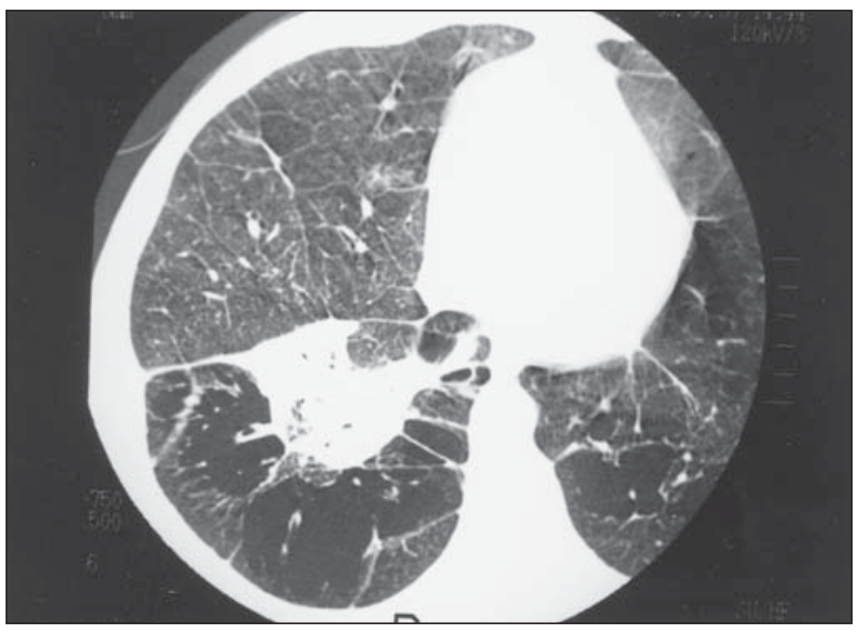

B

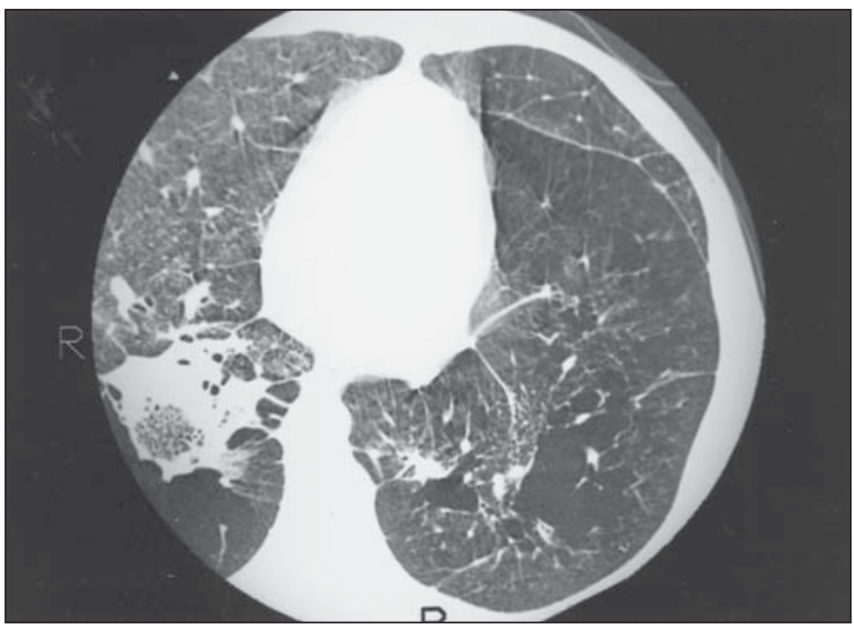

D

Figura 1. Tomografia computadorizada de alta resolução com janelas para parênquima pulmonar evidenciando massas conglomeradas nas regiões póstero-inferiores dos pulmões, com acentuado enfisema adjacente às massas. Observam-se, também, espessamento esparso de septos interlobulares e, nos lobos superiores, pequenos nódulos, áreas de atenuação em vidro fosco e enfisema difuso.

comprimidos, dissolvem o pó resultante em água e injetam esta solução por via endovenosa $^{(\mathbf{4 , 8 , 9 )}}$. Todas essas medicações orais têm em comum a adição de um veículo insolúvel (talco, celulose ou amido), para manter as partículas medicinais juntas e para atuar como lubrificante, impedindo que os comprimidos prendam-se nas máquinas ou entre si durante a sua manufatura $^{(4)}$. Dessa forma, a injeção resulta em êmbolos pulmonares microscópicos ${ }^{(\mathbf{4 , 9 , 1 0 )}}$. As partículas podem migrar para o interstício e causar reação granulomatosa tipo corpo estranho(9).

O talco injetado por via endovenosa é retido no leito vascular pulmonar, que atua como um filtro para as partículas. Assim, na maioria dos casos as lesões ficam limitadas aos pulmões ${ }^{(11)}$. Contudo, casos ra- ros de talcose extrapulmonar têm sido descritos, com lesões no miocárdio, fígado, baço e rins. Nesses casos, as partículas escapam do filtro pulmonar ${ }^{(11)}$.

É interessante que, embora o veículo (talco) seja o mesmo, a reação pulmonar pode ser diferente, de acordo com o tipo de droga injetada. A patogênese do processo não está bem definida, podendo haver diferenças relacionadas à espécie de talco utilizado, sua composição, tamanho das partículas, e também uma ação própria da droga utilizada ${ }^{(\mathbf{1 2})}$.

Cloridrato de metadona injetável foi usado como parte de um programa de desintoxicação de viciados em heroína, no Canadá. Este tratamento pode ser muito demorado, chegando a ser injetados mais de 50.000 comprimidos ao longo de alguns anos. Nesses pacientes observou-se reação granulomatosa difusa, com micronódulos disseminados e formação de massas conglomeradas, especialmente nos campos pulmonares superiores ${ }^{(\mathbf{1 3})}$.

Outros pacientes, em uso de cloridrato de metilfenidato, desenvolveram doença pulmonar obstrutiva grave, do tipo enfisema, com achados radiológicos e tomográficos similares aos encontrados em pacientes com deficiência de alfa-1 antitripsina, e diferentes daqueles observados em outros tipos de uso de drogas endovenosas $^{(\mathbf{9 , 1 0 , 1 4})}$. Esses pacientes desenvolvem enfisema panlobular, mais extenso nas bases, sendo que fibrose intersticial associada foi um achado ausente ou pouco expressivo $^{(\mathbf{9 , 1 4})}$. Em maior ou menor grau, granulomas de talco, infiltrados inflamató- 


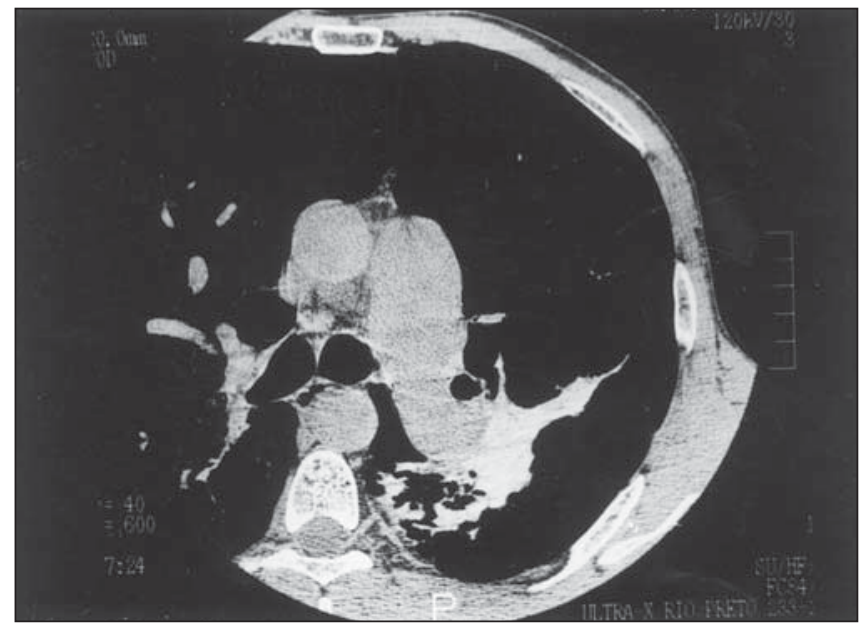

A

Figura 2. Cortes com janelas para mediastino, feitos ao nível das massas conglomeradas, mostrando que elas são heterogêneas, contendo áreas esparsas de elevada atenuação de permeio.

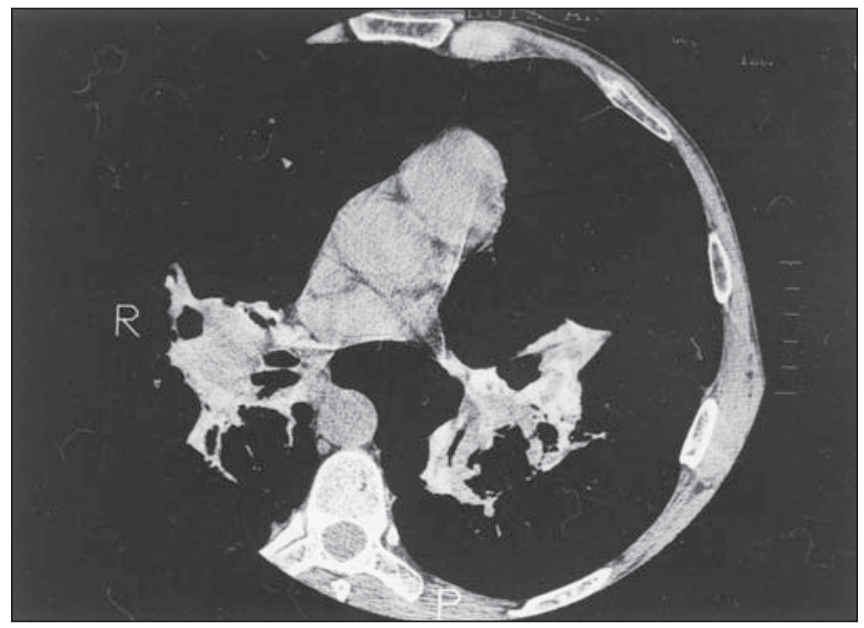

B

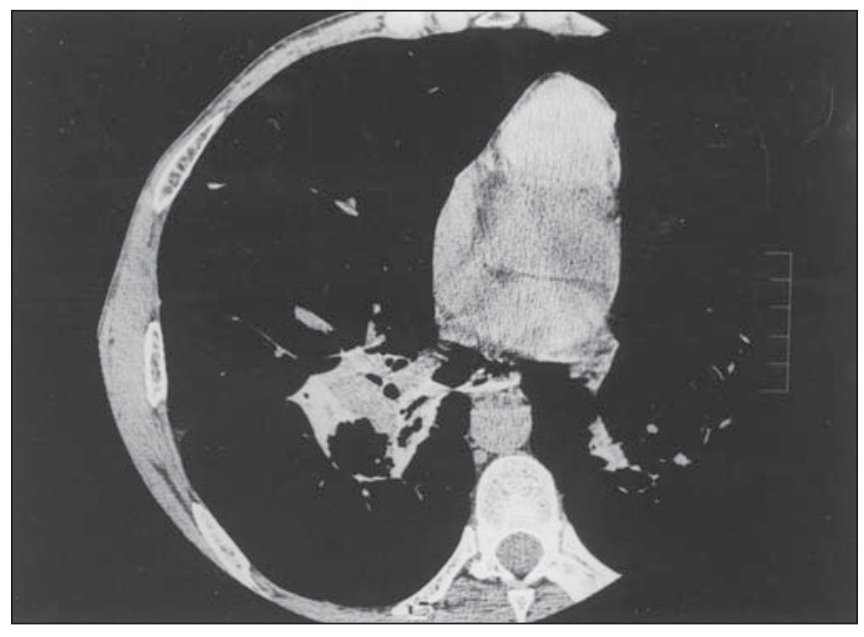

$\operatorname{riados}^{(\mathbf{8 , 1 1})}$. Posteriormente, podem-se desenvolver hipertensão arterial pulmonar e cor pulmonale (10,12). $^{\text {. }}$

O talco causa formação de granulomas, tanto na forma inalada quanto na injeta$\mathrm{da}^{(2)}$. Na patologia, encontra-se basicamente reação inflamatória intersticial, composta por fagócitos, células gigantes multinucleadas tipo corpo estranho, e numerosos cristais birrefringentes, em agulha. Cristais semelhantes a estes, vistos nos septos alveolares, são também encontrados dentro dos espaços alveolares, tanto livres como dentro de macrófagos ${ }^{(\mathbf{3 , 4 , 9 , 1 4})}$. A reação inflamatória pode progredir para fibrose intersticial e enfisema ${ }^{(\mathbf{1 0 , 1 1})}$. Pode haver também comprometimento da via aérea, com bronquite aguda ou crônica ${ }^{(2)}$.

Os granulomas podem confluir, formando áreas de fibrose maciça progressiva, que são compostas por células mononucleares, células gigantes multinucleadas contendo talco, e tecido fibroso relativamente ace$\operatorname{lular}^{(4)}$.

A fibrose maciça progressiva, às vezes, é mais celular, com fibrose de intensidade leve a moderada, lembrando a fibrose dos mineiros de carvão e a dos trabalhadores com caulim. Nesses casos ela é diferente daquela observada nas lesões conglomeradas da silicose $\mathrm{e}^{(\boldsymbol{9})}$.

O diagnóstico histopatológico é estabelecido pelo encontro de fibrose peribrônquica e perivascular, com granulomas tipo corpo estranho não-caseosos, dentro das áreas fibróticas e nos septos alveolares ${ }^{(\mathbf{1 1})}$. O achado característico é a presença de partículas birrefringentes em forma de agulha nas áreas de fibrose, com o uso de luz polarizada $^{(3)}$

Exames de escarro ou de lavado broncoalveolar em geral não fazem o diagnóstico, uma vez que os granulomas estão no interstício $^{(11)}$. Alguns autores ${ }^{(8)}$, contudo, 
relataram o achado de partículas de talco no lavado, mesmo em casos secundários à injeção endovenosa. Os achados laboratoriais podem ser semelhantes aos da sarcoidose $^{(\mathbf{8})}$. O diagnóstico pode também ser feito pelo encontro de cristais de talco em vasos retinianos $^{(\mathbf{4 , 1 0 , 1 3})}$.

As alterações radiológicas iniciais consistem de um padrão micronodular difuso, com pequenos nódulos bem definidos, disseminados $^{(4,7,10,12)}$. Este padrão, causado pelos granulomas, pode ser muito semelhante ao da tuberculose miliar ${ }^{(2,3,11)}$. Com a progressão da doença, os nódulos podem coalescer, resultando em grandes opacidades localizadas nas regiões peri-hilares, semelhantes às vistas na fibrose maciça progressiva da silicose ${ }^{(\mathbf{4 , 7 , 1 0})}$. Na evolução, enfisema grave, com formação de bolhas, pode surgir. Pneumotórax pode complicar o quadro. Em alguns casos, linfonodomegalias estão presentes $^{(7)}$.

Na TCAR podem ser visualizados nódulos, áreas de atenuação em vidro fosco e massas conglomeradas peri-hilares, eventualmente com áreas de alta atenuação no interior, determinadas por deposição de $\operatorname{talco}^{(7)}$.

Ward et al. ${ }^{(9)}$, estudando as tomografias de 12 pacientes com talcose, encontraram quatro padrões principais: micronodulação difusa, massas conglomeradas para-hilares, atenuação em vidro fosco e enfisema. Estes padrões freqüentemente apareceram associados. O enfisema panlobular, de lobos inferiores, predominou nos pacientes que faziam uso de cloridrato de metilfenidato. Os nódulos determinam um aspecto granular fino que, em muitas áreas, são suficientemente numerosos para gerar um padrão de atenuação em vidro fosco. Massas conglomeradas localizadas em regiões peri-hilares, com áreas de alta atenuação (equivalente à do cálcio), podem estar associadas.

$\mathrm{O}$ talco, sem associação com o asbesto, não causa placas pleurais ou diafragmáticas, nem calcificações pleurais ${ }^{(2)}$.

Concluindo, o achado na TCAR de pequenos nódulos centrolobulares, associados a massas conglomeradas heterogêneas, com áreas amorfas de densidade elevada (semelhante à do cálcio) de permeio, com ou sem enfisema panlobular nos lobos inferiores, é altamente sugestivo de talcose pulmonar.

\section{REFERÊNCIAS}

1. Gibbs AE, Pooley FD, Griffiths DM, Mitha R, Craighead JE, Ruttner JR. Talc pneumoconiosis: a pathologic and mineralogic study. Hum Pathol 1992;23:1344-54.

2. Feigin DS. Talc: understanding its manifestations in the chest. AJR 1986;146:295-301.
3. Nam K, Gracey DR. Pulmonary talcosis from cosmetic talcum powder. JAMA 1972;221:492-3.

4. Paré JP, Cote G, Fraser RS. Long-term follow-up of drug abusers with intravenous talcosis. Am Rev Respir Dis 1989;139:233-41.

5. Joshi JM, Kolhe NV, Sundaram P. Pneumoconiosis. Postgrad Med J 1997;73:513-4.

6. Cruthirds TP, Cole FH, Paul RN. Pulmonary talcosis as a result of massive aspiration of baby powder. South Med J 1977;70:626-8.

7. Fraser RS, Müller NL, Colman N, Paré PD. Fraser and Paré's Diagnosis of diseases of the chest. 4th ed. Philadelphia: Saunders, 1999.

8. Farber HW, Fairman RP, Glauser FL. Talc granulomatosis: laboratory findings similar to sarcoidosis. Am Rev Respir Dis 1982;125:258-61.

9. Ward S, Heyneman LE, Reittner P, Kazerooni EA, Godwin JD, Müller NL. Talcosis associated with IV abuse of oral medications: CT findings. AJR 2000; 174:789-93.

10. Padley SPG, Adler BD, Staples CA, Miller RR, Müller NL. Pulmonary talcosis: CT findings in three cases. Radiology 1993;186:125-7.

11. Ben-Haim SA, Ben-Ami H, Edoute Y, Goldstien N, Barzilai D. Talcosis presenting as pulmonary infiltrates in an HIV-positive heroin addict. Chest 1988; 94:656-8.

12. Stern EJ, Frank MS, Schmutz JF, Glenny RW, Schmidt RA, Godwin JD. Panlobular pulmonary emphysema caused by i.v. injection of methylphenidate (Ritalin): findings on chest radiographs and CT scans. AJR 1994;162:555-60.

13. Sieniewicz DJ, Nidecker AC. Conglomerate pulmonary disease: a form of talcosis in intravenous methadone abusers. AJR 1980;135:697-702.

14. Schmidt RA, Glenny RW, Godwin JD, Hampson NB, Cantino ME, Reichenbach DD. Panlobular emphysema in young intravenous Ritalin abusers. Am Rev Respir Dis 1991;143:649-56. 